\title{
InSAR Phase Unwrapping: A Bayesian Approach
}

\author{
José M. B. Dias and José M. N. Leitão \\ Instituto de Telecomunicações \\ Instituto Superior Técnico \\ Torre Norte, Piso 10, Av. Rovisco Pais, \\ 1049-001 Lisboa Portugal \\ E-mail: \{bioucas, jleitao\}@lx.it.pt
}

\begin{abstract}
The paper proposes a Bayesian approach to absolute phase (not simply modulo- $2 \pi$ ) estimation in interferometric aperture radar (InSAR). The observation density is $2 \pi$-periodic and accounts for the interferometric pair decorrelation and the system noise; the a priori probability of the absolute phase is modeled by a compound Gauss Markov random field (CGMRF). To compute the absolute phase estimate we propose an iterative scheme aiming at the computation of the maximum a posteriori probability (MAP) estimate. Each iteration embodies a discrete optimization step (Z-step), implemented by network programming techniques, and an iterative conditional modes (ICM) step ( $\pi$-step). According to the terms $\mathbb{Z}$-step and $\pi$-step, we term our algorithm $\mathbb{Z} \pi \mathrm{M}$, where the letter $\mathrm{M}$ stands for maximization. Experimental results, comparing the proposed algorithm with classical approaches, illustrates the effectiveness of the $\mathbb{Z} \pi \mathrm{M}$ algorithm.
\end{abstract}

\section{INTRODUCTION}

In a SAR system, as in any coherent system, only noisy versions of the principal phase values (modulo- $2 \pi$ ) are available, as they are computed from the argument of the received wave. However, in InSAR applications, the objective is the estimation of the absolute phase (phase unwrapping in the InSAR jargon), and not simply its modulo- $2 \pi$.

Classical phase unwrapping methods are either of path following type or of minimum-norm type [1]. In the path following schemes phase is unwrapped through selected image paths. In the presence of discontinuities or noise, different paths between two points may lead to different absolute phase values. To resolve or mitigate theses inconsistencies, heuristic rules are applied to provide path independent integration. The minimum-norm phase unwrapping methods cast the unwrapping problem into the minimization of a $L^{p}$ norm.

The mainstream of absolute phase estimation research in InSAR takes a two step approach: in the first step the so-called interferogram (principal phase values) is inferred from noisy InSAR image pairs; in the second step the phase is unwrapped by determining the $2 \pi$ multiples. The book [1] and the algorithms therein presented and compared are representative of this approach. Along this paper we use the term unwrapping to designate the latter step.

This work was supported by the Fundação para a Ciência e Tecnologia, under the project POSI/34071/CPS/2000.
The smoothness step applied by most classical phase unwrapping algorithms assumes that phase is practically constant within small windows, this not being true for high phase rate regions. Therefore in these regions the smooth principal phase values exhibits inconsistency and are a source of error in any unwrapping algorithm that does not take them into account.

\section{A. Proposed Approach}

We adopt the Bayesian viewpoint. The likelihood function, which models the observation mechanism given the absolute phase, is $2 \pi$-periodic and accounts for the interferometric pair decorrelation and the system noise. The a priori probability of the absolute phase is modeled by a first order compound Gauss Markov random field (CGMRF) [2]. The CGMRFs are suited to piecewise smooth fields, and, therefore, to model discontinuities between smooth regions.

Papers [3], and [4] have also adopted the Bayesian approach, where the prior is a first order causal GMRF. Taking advantage of this prior and using the reduced order model (ROM) approximation of the GMRF, the absolute phase is estimated with a nonlinear recursive filtering technique. Compared with the present approach, the main difference concerns the prior: we use a first order noncausal CGMRF prior. In terms of estimation, the noncausal prior has implicit a batch perspective, whereas the causal prior has implicit a recursive perspective.

To compute the MAP estimate we propose an iterative procedure made of two steps per iteration: the first step, termed $\mathbb{Z}$-step, maximizes the posterior density with respect to the field of $2 \pi$ phase multiples; the second step, termed $\pi$-step, maximizes the posterior density with respect to the phase principal values. According to the terms $\mathbb{Z}$-step and $\pi$-step, we term our algorithm $\mathbb{Z} \pi \mathrm{M}$, where the letter $\mathrm{M}$ stands for maximization.

\section{Proposed Models}

\section{A. Observation Model}

Let $x_{1}$ and $x_{2}$ be the complex amplitudes read by two SAR sensors from a given site. The height $h$ of a given terrain element is a function $g(\phi)$ of the phase $\phi=\phi_{1}-\phi_{2}$, 
where $\phi_{1}$ and $\phi_{2}$ are the propagation path phases associated to sensor 1 and sensor 2, respectively. Phase $\phi$ is to be inferred from $x_{1}$ and $x_{2}$. These complex amplitudes are given by

$$
\begin{aligned}
& x_{1}=z_{1} e^{-j \phi_{1}}+n_{1} \\
& x_{2}=z_{2} e^{-j \phi_{2}}+n_{2},
\end{aligned}
$$

where $z_{1}$ and $z_{2}$ are the complex amplitudes originated by the scatterers illuminated by apertures 1 and 2, respectively, and $n_{1}$ and $n_{2}$ are the electronic noise of sensor 1 and sensor 2 , respectively.

Assuming that the surface being illuminated is rough compared to the wavelength, that there is no strong specular reflectors, and that there is a large number of scatterers per resolution cell, then the complex amplitude $z_{1}$ is complex zero-mean circular Gaussian distributed [5, ch. 5]. Noises $n_{1}$ and $n_{2}$ are also independent (correspond to different sensors) and complex zero-mean circular Gaussian distributed. Furthermore, we assume that $n_{1}$ and $n_{2}$ are independent of $z_{1}$ and $z_{2}$. Complex amplitudes $z_{1}$ and $z_{2}$ are different due to decorrelation (spatial, temporal, and geometric) and focusing errors.

Defining $\theta^{2} \equiv E\left[\left|z_{1}\right|^{2}\right]=E\left[\left|z_{2}\right|^{2}\right], \alpha \equiv E\left[z_{1} z_{2}^{*}\right] / \theta^{2}, x \equiv$ $\left[\begin{array}{ll}x_{1} & x_{2}\end{array}\right]^{T}, \sigma_{n}^{2} \equiv E\left[\left|n_{1}\right|^{2}\right]$, and assuming that $E\left[\left|n_{1}\right|^{2}\right]=$ $E\left[\left|n_{2}\right|^{2}\right]$, the probability density function ${ }^{1}$ of $x$ is $[6, \mathrm{ch}$. 3]

$$
p_{x \mid \phi}(x \mid \phi)=\frac{1}{\pi^{2}|\mathbf{Q}|} e^{-x^{H} \mathbf{Q}^{-1} x},
$$

where $\mathbf{Q} \equiv E\left[x x^{H}\right]$ is given by

$$
\mathbf{Q}=\left[\begin{array}{cc}
\theta^{2}+\sigma_{n}^{2} & \alpha \theta^{2} e^{j \phi} \\
\alpha \theta^{2} e^{-j \phi} & \theta^{2}+\sigma_{n}^{2}
\end{array}\right]
$$

Developing the quadratic form in (3), one is led to

$$
p_{x \mid \phi}(x \mid \phi)=c e^{\lambda \cos (\phi-\eta)},
$$

where $c=c(x, \theta, \alpha)$ and

$$
\begin{aligned}
\eta & =\arg \left(x_{1} x_{2}^{*}\right) \\
\lambda & =\frac{2 \alpha \theta^{2}\left|x_{1} x_{2}\right|}{|\mathbf{Q}|} .
\end{aligned}
$$

The likelihood function $p_{x \mid \phi}(x \mid \phi)$ is $2 \pi$-periodic with respect to $\phi$ with maxima at $\phi=2 \pi k+\eta$, for $k \in \mathbb{Z}(\mathbb{Z}$ denotes the integer set). Parameter $\eta$ is a maximum likelihood estimate of $\phi$. The peakiness of (5) about $2 \pi k+\eta$, controlled by parameter $\lambda$, is an indication, in a statistical sense, of how trustful data is.

Let $\boldsymbol{\phi} \equiv\left\{\phi_{i j} \mid(i, j) \in Z\right\}$ and $\mathbf{x} \equiv\left\{x_{i j} \mid(i, j) \in Z_{o}\right\}$ denote the absolute phase and complex amplitude associated to sites $Z \equiv\{(i, j) \mid i, j=1, \ldots, N\}$ (we assume without

\footnotetext{
${ }^{1}$ For compactness, lowercase letters will denote random variables and their values as well.
}

lack of generality that images are squared) and $Z_{o} \subset Z$, respectively. Assuming that the components of $\mathbf{x}$ are conditionally independent, then

$$
p_{\mathbf{x} \mid \phi}(\mathbf{x} \mid \phi)=\prod_{i j \in Z_{o}} p_{x_{i j} \mid \phi_{i j}}\left(x_{i j} \mid \phi_{i j}\right)
$$

As stated before, we assume that the observation set of sites $Z_{0}$ is a subset of the absolute phase sites $Z$. Sites $Z-Z_{0}$ are either not observed or belong to inconsistent phase regions.

\section{B. Prior Model}

Image $\phi$ is assumed to be piecewise smooth, with abrupt variations between neighboring regions. This variations are due to undersampling in areas with high fringe rates, mainly due to the presence of layover phenomena and/or abrupt feature or objects. Independently of its origin, discontinuities of the absolute phase $\phi$ are the principal source of error in any unwrapping algorithm that does not take them into account.

Gauss-Markov random fields [7], [8] are both mathematically and computationally suitable for representing local interactions, and particularly continuity between neighboring pixels. However, the continuity constraint must be discarded for those pixels in the neighborhood of discontinuities. For this purpose we take the first order noncausal CGMRF [2], whose density is

$$
p_{\phi \mid \mathbf{l}}(\phi \mid \boldsymbol{l}) \propto \exp \left\{-\frac{\mu}{2} \sum_{i j \in Z_{1}}\left(\Delta \phi_{i j}^{h}\right)^{2} \bar{v}_{i j}+\left(\Delta \phi_{i j}^{v}\right)^{2} \bar{h}_{i j}\right\},
$$

where $\mathbf{l} \equiv\left\{v_{i j}, h_{i j} \mid(i, j) \in Z\right\}$ is the so-called line field process, $\bar{v}_{i j} \equiv\left(1-v_{i j}\right), \bar{h}_{i j} \equiv\left(1-h_{i j}\right), \Delta \phi_{i j}^{h} \equiv$ $\left(\phi_{i j}-\phi_{i, j-1}\right), \Delta \phi_{i j}^{v} \equiv\left(\phi_{i j}-\phi_{i-1, j}\right), Z_{1} \equiv\{(i, j) \mid i, j=$ $2, \ldots, N\}$, and $\mu^{-1}$ means the variance of increments $\Delta \phi_{i j}^{h}$ and $\Delta \phi_{i j}^{v}$. Variables $v_{i j}, h_{i j} \in\{0,1\}$ serve the purpose of signaling discontinuities. Notice that the continuity constraint between sites $(i, j)$ and $(i, j-1)$ is removed if variable $v_{i j}$ is set to one; similarly, the continuity constraint between sites $(i, j)$ and $(i-1, j)$ is removed if variable $v_{i j}$ is set to one.

\section{Posterior Density}

Consider that the line field process $\mathbf{l}$ is known. Invoking the Bayes rule, and noting that $p_{\mathbf{x} \mid \phi, \mathbf{l}}(\mathbf{x} \mid \phi, \mathbf{l})=p_{\mathbf{x} \mid \phi}(\mathbf{x} \mid \phi)$, we obtain the posterior probability density function of $\phi$, given $(\mathbf{x}, \mathbf{l})$, as

$$
p_{\phi \mid \mathbf{x}, \mathbf{l}}(\phi \mid \mathbf{x}, \mathbf{l}) \propto p_{\mathbf{x} \mid \phi}(\mathbf{x} \mid \boldsymbol{\phi}) p_{\phi \mid \mathbf{l}}(\boldsymbol{\phi} \mid \mathbf{l}),
$$


where the factors not depending on $\phi$ were discarded. Introducing (8) and (9) into (10), we obtain

$$
\begin{gathered}
\sum_{p_{\phi \mid \mathbf{x}, \mathbf{l}}(\phi \mid \mathbf{x}, \mathbf{l})} \propto e^{i j \in Z_{0}} \lambda_{i j} \cos \left(\phi_{i j}-\eta_{i j}\right) \\
\times e^{-\frac{\mu}{2} \sum_{i j \in Z_{1}}\left(\left(\Delta \phi_{i j}^{h}\right)^{2} \bar{v}_{i j}+\left(\Delta \phi_{i j}^{v}\right)^{2} \bar{h}_{i j}\right)}
\end{gathered}
$$

The posterior distribution (11) is assumed to contain all information one needs to compute the absolute phase estimate $\widehat{\phi}$.

\section{Estimation Procedure}

The MAP criterion is adopted for computing $\widehat{\phi}$. Accordingly

$$
\widehat{\phi}_{M A P}=\arg \max _{\phi} p_{\phi \mid \mathbf{x}, \mathbf{l}}(\phi \mid \mathbf{x}, \mathbf{l}) .
$$

Due to the periodic structure of $p_{x \mid \phi}(x \mid \phi)$, computing the MAP solution leads to a huge non-convex optimization problem, with unbearable computation burden. Instead of computing the exact estimate $\widehat{\phi}_{M A P}$, we resort to a suboptimal scheme that delivers nearly optimal estimates, with a far less computational load.

Consider, for a while, that $Z_{o}=Z$, i.e., all sites are observed. Let the image $\phi_{i j}$ be uniquely decomposed as

$$
\phi_{i j}=\psi_{i j}+2 \pi k_{i j},
$$

where $k_{i j}=\left\lfloor\left(\phi_{i j}+\pi\right) /(2 \pi)\right\rfloor \in \mathbb{Z}$ is the so-called wrapcount component of $\phi_{i j}$ and $\psi_{i j} \in[-\pi, \pi$ [ is the principal value of $\phi_{i j}$. The MAP estimate (12) can be rewritten in terms of $\boldsymbol{\psi} \equiv\left\{\psi_{i j} \mid(i, j) \in Z\right\}$ and $\mathbf{k} \equiv\left\{k_{i j} \mid(i, j) \in Z\right\}$ as

$$
\begin{aligned}
& \left(\widehat{\boldsymbol{\psi}}_{M A P}, \widehat{\mathbf{k}}_{M A P}\right)= \\
& \quad=\arg \max _{\psi, \mathbf{k}} p_{\phi \mid \mathbf{x}, \mathbf{l}}(\boldsymbol{\psi}+2 \pi \mathbf{k} \mid \mathbf{x}, \mathbf{l}) \\
& \quad=\arg \left\{\max _{\boldsymbol{\psi}}\left\{\max _{\mathbf{k}} p_{\phi \mid \mathbf{x}, \mathbf{l}}(\boldsymbol{\psi}+2 \pi \mathbf{k} \mid \mathbf{x}, \mathbf{l})\right\}\right\} .
\end{aligned}
$$

Instead of computing (15), we propose a procedure that successively and iteratively maximizes $p_{\phi \mid \mathbf{x}, \mathbf{l}}(\boldsymbol{\psi}+2 \pi \mathbf{k} \mid \mathbf{x}, \mathbf{l})$ with respect to $\mathbf{k} \in \mathbb{Z}^{N^{2}}$ and $\boldsymbol{\psi} \in\left[-\pi, \pi\left[{ }^{N^{2}}\right.\right.$. We term this maximization on sets $\mathbb{Z}$ and $[-\pi, \pi[$ as the $\mathbb{Z} \pi \mathrm{M}$ algorithm; Fig. 1 shows the corresponding pseudo-code.

The unwrapping step (16) finds the maximum of the posterior density $p_{\phi \mid \mathbf{x}, \mathbf{l}}(\phi \mid \mathbf{x}, \mathbf{l})$ on a mesh obtained by discretizing each coordinate $\phi_{i j}$ according to (13). The first estimate $\widehat{\mathbf{k}}^{(1)}$ delivered by the unwrapping step is based on the maximum likelihood estimate $\boldsymbol{\eta} \equiv\left\{\eta_{i j} \mid(i, j) \in Z\right\}$. Smoothing is next implemented by the $\pi$-step (17). This is in contrast with the scheme followed by most phase unwrapping algorithms, where the phase is estimated with
Initialization: $\widehat{\psi}^{(0)}=\eta$

For $t=1,2, \ldots$,

Unwrapping step:

$$
\widehat{\mathbf{k}}^{(t)}=\arg \max _{\mathbf{k}} p_{\phi \mid \mathbf{x}, \mathbf{l}}\left(\boldsymbol{\psi}^{(t-1)}+2 \pi \mathbf{k} \mid \mathbf{x}, \mathbf{l}\right)
$$

Smoothing step:

$$
\widehat{\boldsymbol{\psi}}^{(t)}=\arg \max _{\psi} p_{\boldsymbol{\phi} \mid \mathbf{x}, \mathbf{l}}\left(\boldsymbol{\psi}+2 \pi \mathbf{k}^{(t)} \mid \mathbf{x}, \mathbf{l}\right)
$$

Termination test:

$$
\begin{aligned}
& \text { If } \quad\left[p_{\phi \mid \mathbf{x}, \mathbf{l}}\left(\widehat{\boldsymbol{\phi}}^{(t)} \mid \mathbf{x}, \mathbf{l}\right)-p_{\phi \mid \mathbf{x}, \mathbf{l}}\left(\widehat{\boldsymbol{\phi}}^{(t-1)} \mid \mathbf{x}, \mathbf{l}\right)\right]<\xi \\
& \text { break loop for }
\end{aligned}
$$

Fig. 1. $\mathbb{Z} \pi \mathbf{M}$ Algorithm.

basis on on a smooth version of $\boldsymbol{\eta}$, under the assumption that the phase $\phi$ is constant within windows of given size. This assumption leads to strong errors in areas of high phase rate.

\section{A. $\mathbb{Z}$-Step}

Since the logarithm is strictly increasing and $\cos \left(\psi_{i j}+\right.$ $2 \pi k_{i j}-\eta_{i j}$ ) does not depend on $k_{i j}$, solving the maximization step (16) is equivalent to solve

$$
\widehat{\mathbf{k}}=\arg \min _{\mathbf{k}} E(\mathbf{k} \mid \boldsymbol{\psi})
$$

where the energy $E(\mathbf{k} \mid \psi)$ is given by

$$
E(\mathbf{k} \mid \psi) \equiv \sum_{i j \in Z_{1}}\left(\Delta \phi_{i j}^{h}\right)^{2} \bar{v}_{i j}+\left(\Delta \phi_{i j}^{v}\right)^{2} \bar{h}_{i j}
$$

Minimization (18) is a nearest lattice vector problem and it is NP-hard [9]. In our case, energy $E(\mathbf{k} \mid \psi)$ is a sum of quadratic functions of $\left(k_{i j}-k_{i-1, j}\right)$ and $\left(k_{i j}-k_{i, j-1}\right)$. This is a special case of a nearest lattice vector problem, for which we propose a network programming algorithm that finds the exact solution in polynomial time. The algorithm is inspired in the Flyn's minimum discontinuity approach [10], which minimizes the sum of $\left|\left\lfloor\Delta \phi_{i j}^{h}+\pi\right\rfloor\right|$ and $\left|\left\lfloor\Delta \phi_{i j}^{v}+\pi\right\rfloor\right|$, where $\lfloor x\rfloor$ denotes the hightest integer lower than $x$. Flyn's objective function is, therefore, quite different from ours. However, both objective functions are the sum of first order click potentials depending only on $\Delta \phi_{i j}^{h}$, and $\Delta \phi_{i j}^{v}$. This structural similarity allows us to adapt the above ideas to our problem.

The detailed description of the $\mathbb{Z}$-step are out of the scope of this paper and can be found in [11]. 


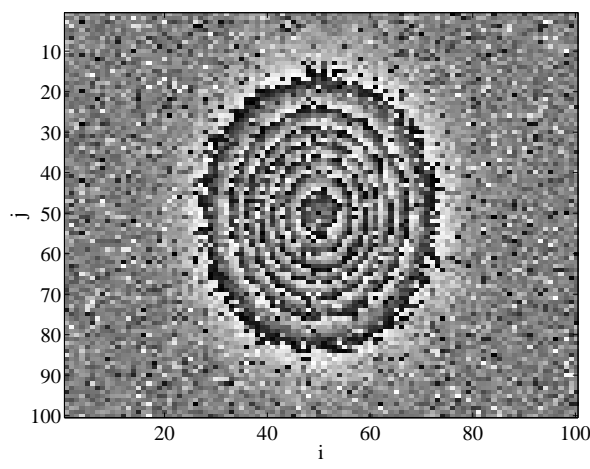

Fig. 2. Interfergram ( $\boldsymbol{\eta}$-image) of a Gaussian elevation of height $14 \pi$ rad and standard deviations $\sigma_{i}=10$ and $\sigma_{j}=15$ pixels. The correlation coefficient of the associated InSAR pair is $\alpha=0.8$.

\section{B. Smoothing Step}

The smoothing step (17) amounts to compute $\widehat{\boldsymbol{\psi}}$ given by

$$
\begin{aligned}
\widehat{\boldsymbol{\psi}}= & \arg \max _{\boldsymbol{\psi} \in\left[-\pi, \pi\left[N^{2}\right.\right.} \sum_{i j \in Z} \lambda_{i j} \cos \left(\phi_{i j}-\eta_{i j}\right) \\
& -\sum_{i j \in Z_{1}} \frac{\mu}{2}\left(\left(\Delta \phi_{i j}^{h}\right)^{2} \bar{v}_{i j}+\left(\Delta \phi_{i j}^{v}\right)^{2} \bar{h}_{i j}\right),
\end{aligned}
$$

where $\phi_{i j}=2 \pi k_{i j}+\psi_{i j}$. The function to be maximized in (20) is not convex due to terms $\lambda_{i j} \cos \left(\phi_{i j}-\eta_{i j}\right)$. Computing $\widehat{\psi}$ is therefore a hard problem. Herein, we adopt the ICM approach [7], which, in spite of being suboptimal, yields good results for the problem at hand.

ICM is a coordinatewise ascent technique where all coordinates are visited according to a given schedule. After some simple algebraic manipulation of the objective function (20), we conclude that its maximum with respect to $\psi_{i j}$ is given by

$$
\widehat{\psi}_{i j}=\arg \max _{\psi_{i j} \in[-\pi, \pi[}\left\{\beta_{i j} \cos \left(\psi_{i j}-\eta_{i j}\right)-\left(\psi_{i j}-\bar{\psi}_{i j}\right)^{2}\right\},
$$

with $\beta_{i j}=2 \frac{\lambda_{i j}}{\mu \bar{l}_{i j}}, \bar{\psi}_{i j}=\bar{\phi}_{i j}-2 \pi k_{i j}$, where $\bar{l}_{i j}$ is the sum of signaled discontinuities in the neighborhood of site $(i, j)$ and $\bar{\phi}_{i j}$ is the mean phase of first order neighbors not signaled with discontinuities.

There are no closed form solutions for maximization (21), since it involves transcendent and power functions. We compute $\widehat{\psi}_{i j}$ using a simple two-resolution numeric method. First we search $\widehat{\psi}_{i j}$ in the set $\{\pi i / M \mid i=$ $-M, \ldots, M-1\}$. Next we refine the search by using the set $\left\{\pi i_{0} / M+\pi i / M^{2} \mid i=-M, \ldots, M-1\right\}$, where $\pi i_{0} / M$ is the result of the first search. We have used $M=20$, which leads to the maximum error of $\pi /(20)^{2}$.

\section{RESUlts}

The algorithm presented in the previous sections is now applied to synthetic data. Fig. 2 displays the interfero-
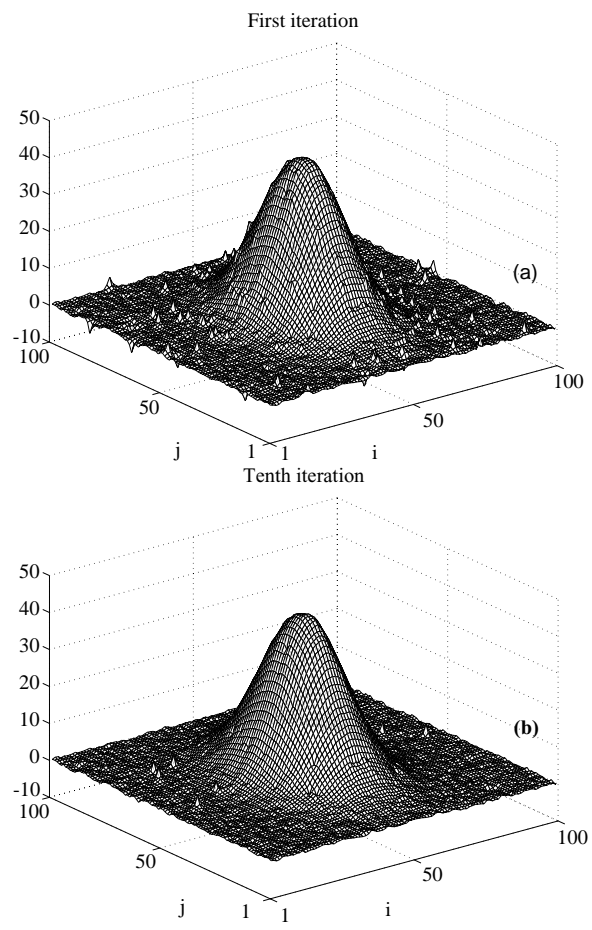

Fig. 3. Phase estimate $\widehat{\phi}^{(t)}$; (a) $t=1$; (b) $t=10$.

gram $\left(\boldsymbol{\eta}=\left\{\eta_{i j}\right\}\right.$ image) obtained with parameters $\theta=1$, $\alpha=0.8$ and $\sigma_{n}=0$. The absolute phase image $\phi$ is a Gaussian elevation of height $14 \pi$ rad and standard deviations $\sigma_{i}=10$ and $\sigma_{j}=15$ pixels. The magnitude of the phase difference $\phi_{i, j+1}-\phi_{i j}$ takes the maximum value of 2.5 and is greater than 2 in many sites. On the other hand a correlation coefficient of $\alpha=0.8$ implies a standard deviation of the maximum likelihood estimate $\eta_{i j}$ of 0.91. This figure is computed with basis on the density of $\eta$ obtained from the joint density (3). In these conditions, the task of absolute phase estimation is extremely hard, as the interferogram exhibits a large number of inconsistencies; i.e., the observed image $\boldsymbol{\eta}$ is not consistent with the assumption of absolute phase differences less than $\pi$ in a large number of sites. In the unwrapping jargon we say that the interferogram has a lot of residues.

The smoothness parameter was set to $\mu=1 / 0.8^{2}$, thus modeling phase images with phase differences (horizontal and vertical) of standard deviation 0.8 . This value is too large for most of the true absolute phase image $\phi$ and too small for sites in the neighborhood of sites $(i=-45, j=$ $50)$ and $(i=55, j=50)$ (where the magnitude of the phase difference has its largest value). Nevertheless, the $\mathbb{Z} \pi \mathrm{M}$ algorithm yields good results as it can be read from Fig. 3; Fig. 3(a) shows the phase estimate $\widehat{\phi}^{(1)}$ and Fig. 3(b) shows the phase estimate $\widehat{\phi}^{(10)}$.

To rank $\mathbb{Z} \pi \mathrm{M}$ algorithm, we have applied the following phase unwrapping algorithms to the present problem: 
TABLE I

$L^{2}$ NORM OF THE ESTIMATION ERRORS OF $\mathbb{Z} \pi$ M AND OF CLASSICAL UNWRAPPING ALGORITHMS. THE LEFT COLUMN PLOTS RESUltS BASED OF THE THE MAXIMUM LIKELIHOOD ESTIMATE OF $\boldsymbol{\eta}$ USING A $3 \times 3$ RECTANGULAR WINDOW; THE RIGHT COLUMN PLOTS RESULTS BASED ON THE NON-SMOOTH $\boldsymbol{\eta}$ GIVEN BY (6).

\begin{tabular}{l|ll}
\hline \multirow{2}{*}{ Algorithm } & \multicolumn{2}{|c}{$\|\widehat{\boldsymbol{\phi}}-\boldsymbol{\phi}\|^{2}$} \\
\cline { 2 - 3 } & Smooth $\boldsymbol{\eta}$ & Non-smooth $\boldsymbol{\eta}$ \\
\hline $\mathbb{Z} \pi \mathrm{M}$ & - & 0.1 \\
GBC & 48.0 & 7.0 \\
QG & 10.0 & 2.2 \\
MC & 40.8 & 28.6 \\
FMD & 22.4 & 3.4 \\
WLS & 8.8 & 3.5 \\
L0N & 24.1 & 2.6
\end{tabular}

- Path following type: Golstein's branch cut (GBC); quality guided (QG); and mask cut (MC)

- Minimum norm type: Flyn's minimum discontinuity (FMD); weighted least-square (WLS); and $L^{0}$ norm (LON).

Path following and minimum norm algorithms were implemented with the code supplied in the book [1], using the following settings: GBC (-dipole yes); QG, MC, (mode min_var -tsize 3); and WLS (-mode min_var -tsize 3 , -thresh yes). The unweighted versions of the FMD and L0N algorithms have been used.

Table I displays the $L^{2}$ norm of the estimation error $\|\widehat{\phi}-\phi\|^{2}$ for each of the classic algorithm referred above. Results on the left column area based on the maximum likelihood estimate of $\boldsymbol{\eta}$, using a $3 \times 3$ rectangular window. Results on the right column are based on the interferogram $\boldsymbol{\eta}$ without any smoothing. Apart from the proposed $\mathbb{Z} \pi \mathrm{M}$ scheme, all the algorithms have produced poor results, some of them catastrophic.

\section{Concluding Remarks}

The paper presented an effective algorithm to absolute phase estimation in InSAR applications. The Bayesian standpoint was adopted. The likelihood function, which models the observation mechanism given the absolute phase, is $2 \pi$-periodic and accounts for interferometric pair decorrelation and system noise. The a priori probability of the absolute phase is modelled by a noncausal first order compound Gauss Markov random field (CGMRF). This prior is suited to piecewise smooth fields, in the sense that it enforces smoothness, in a statistical sense, between neighboring sites not split by discontinuities. The adopted framework also models incomplete data observations.

To compute the absolute phase estimate we adopted the maximum a posteriori (MAP) criterion. We derived a suboptimal iterative procedure consisting of two steps per iteration: the first step, termed $\mathbb{Z}$-step, maximimizes the posterior density with respect to the $2 \pi$ phase multiples; the second step, termed $\pi$-step, maximize the posterior density with respect to the phase principal values. The $\mathbb{Z}$ step is a discrete optimization problem solved exactly by network programing techniques inspired by Flyn's minimum discontinuity algorithm [10]. The $\pi$-step is a continuous optimization problem solved approximately by the iterated conditional modes (ICM) procedure. We named the proposed algorithm $\mathbb{Z} \pi \mathrm{M}$, where the letter $M$ stands for maximization.

To evaluate the performance of the $\mathbb{Z} \pi \mathrm{M}$ algorithm, we compared $\mathbb{Z} \pi \mathrm{M}$ estimates with those provided by path following and minimum-norm schemes, namely the Golstein's branch cut, the quality guided, the Flyn's minimum discontinuity, the weighted least-square, and the $L^{0}$ norm. In the studied example, the proposed algorithm yields the best estimate.

\section{REFERENCES}

[1] D. Ghiglia and M. Pritt, Two-Dimentional Phase Unwrapping. Theory, Algorithms, and Software, John Wiley \& Sons, New York, 1998.

[2] F. Jeng and J. Woods, "Compound Gauss-Markov random fields for image processing," in Digital Image Restoration, A.Katsaggelos, Ed., pp. 89-108. Springer Verlag, 1991.

[3] J. Leitão and M. Figueiredo, "Absolute phase image reconstruction: A stochastic nonlinear filtering approach," IEEE Transactions on Image Processing, vol. 7, no. 6, pp. 868-882, June 1997

[4] J. Dias and J. Leitão, "Simultaneous phase unwrapping and speckle smoothing in SAR images: A stochastic nonlinear filtering approach," in EUSAR'98 European Conference on Synthetic Aperture Radar, Friedrichshafen, May 1998, pp. 373-377.

[5] C. Jakowatz, D. Wahl, P. Eichel, D. Ghiglia, and P. Thompson, Spotlight-Mode Synthetic Aperture Radar: A Signal Processing Approach, Kluwer Academic Publishers, Boston, 1996.

[6] K. S. Miller, Complex Stochastic Processes. An Introduction to Theory and Applications, Addison-Wesley Publishing Co., London, 1974.

[7] J. Besag, "On the statistical analysis of dirty pictures," Journal of the Royal Statistical Society B, vol. 48, no. 3, pp. 259-302, 1986.

[8] S. Geman and D. Geman, "Stochastic relaxation, Gibbs distribution and the Bayesian restoration of images," IEEE Transactions on Pattern Analysis and Machine Intelligence, vol. PAMI-6, no. 6, pp. 721-741, November 1984.

[9] M. Grotschel, L. Lovasz, and A. Schrijver, Beometric Algorithms and Combinatorial Optimization, Algorithms and Combinatorics. Springer-Verlag, New York, 1988.

[10] T. Flynn, "Two-dimensional phase unwrapping with minimum weighted discontinuity," Journal of the Optical Society of America A, vol. 14, no. 10, pp. 2692-2701, 1997.

[11] J. Dias and J. Leitão, "The $Z \pi M$ algorithm for interferometric image reconstruction in sar/sas," IEEE Transactions on Image Processing, 2001, Submitted. 